Research Journal of Applied Sciences 6 (7-12): 457-463, 2011

ISSN: $1815-932 \mathrm{X}$

(C) Medwell Journals, 2011

\title{
An Appraisal Study of Power-Line Communication (PLC) and It's Application
}

\author{
Balogun Bashiru Bolaji and Suleiman Kamaldeen Olasunkanmi \\ Department of Pure and Applied Physics, \\ Ladoke Akintola University of Technology, Ogbomoso, Oyo State, Nigeria
}

\begin{abstract}
The communication flow of today is very high. Many applications are operating at high speed in which a fixed connection is often preferred. If the power utilities could supply communication signals over the power-line to the consumers, this could make a tremendous breakthrough in communications. Every household would then be connected at any time and services would be provided at real-time. Using the power-line as a communication medium, communication could also be cost-effective, compared to other systems because it uses an existing infrastructure, wires exists to every household which are connected to the power-line network. In recent times, the deregulated market has forced the power utilities to explore new markets to find new business opportunities which have increased the research in power-line communications the last decade. Researchers have initially focused on providing services related to power distribution such as load control, meter reading, tariff control, remote control and smart homes. These value-added services would open up new markets for the power utilities and hence increase the profit. The moderate demands of these applications make it easier to obtain reliable communication. Firstly, the information bit rate is low; secondly, they do not require real-time performance. During the last years the use of Internet has increased. If it would be possible to supply this kind of network communication over the power-line, the utilities could also become communication providers, a rapidly growing market. On the contrary to power related applications, network communications require very high bit rates and in some cases real-time responses are needed (such as video and TV). This complicates the design of a communication system which has been the focus of many researchers in recent times. Systems under trial exist today that claim a bit rate of $1 \mathrm{Mb} \mathrm{sec}^{-1}$ but most commercially available systems use low bit rates, about $10-100 \mathrm{~kb} \mathrm{sec}^{-1}$ and provides low-demanding services such as meter reading.
\end{abstract}

Key words: Communication, power-line, real time, bit rate, infrastructure, Nigeria

\section{INTRODUCTION}

A modern electric power system consists of six main components, these are the power station, a set of transformers to raise the generated power to the high voltages used on the transmission lines, the transmission lines, the substations at which the power is stepped down to the voltage on the distribution lines, the distribution lines and the transformers that lower the distributed voltage to the level used by the consumer's equipment. Power line communication or Power Line Carrier (PLC) also known as Power line Digital Subscriber Line (PDSL), mains communication, Power Line Telecom (PLT) or Power Line Networking (PLN) is a system that involves carrying data (signals) on a conductor also used for electric power transmission. Broadband over Power
Lines (BPL) uses PLC by sending and receiving radio signals over power lines to provide access to the Internet. Electrical power is transmitted over high voltage transmission lines, distributed over medium voltage and used inside buildings at lower voltages. The mains wiring in the average home can support a variety of frequencies. As electricity uses $50 / 60 \mathrm{~Hz}$ signals, extra data can be transported along the same wiring at much higher frequencies without causing any interference. Power-line communications can be applied at each stage. Most Power Line Communication (PLC) technologies limit themselves to one set of wires (for example, premises wiring) but some can cross between two levels (for example, both the distribution network and premises wiring). All power line communications systems operate by impressing a modulated carrier signal on the wiring

Corresponding Author: Balogun Bashiru Bolaji, Department of Pure and Applied Physics, Ladoke Akintola University of Technology, Ogbomoso, Oyo State, Nigeria 
system. Different types of power line communications use different frequency bands, depending on the signal transmission characteristics of the power wiring used.

Since, the power wiring system was originally intended for transmission of $\mathrm{AC}$ power, the power wire circuits have only a limited ability to carry higher frequencies. The propagation problem is a limiting factor for each type of power line communications. Data rates over a power line communication system vary widely. Low-frequency (about 100-200 kHz) carriers impressed on high-voltage transmission lines may carry one or two analog voice circuits or telemetry and control circuits with an equivalent data rate of a few hundred bits per second however, these circuits may be many miles $(\mathrm{km})$ long. Higher data rates generally imply shorter ranges; a local area network operating at millions of bits per second may only cover one floor of an office building but eliminates installation of dedicated network cabling.

\section{POWER LINE CARRIER SYSTEMS}

Power line carrier systems have long been a favorite at many utilities because it allows them to reliably move data over an infrastructure that they control. Many technologies are capable of performing multiple applications. For example, a communication system bought initially for automatic meter reading can sometimes also be used for load control or for demand response applications.

PLC is one of the technologies used in the automatic meter reading industry. Both one-way and two-way systems have been successfully used for decades. Interest in this application has grown substantially in recent history not so much because there is an interest in automating a manual process but because there is an interest in obtaining fresh data from all metered points in order to better control and operate the system. PLC is one of the technologies being used in Advanced Metering Infrastructure (AMI) systems.

\section{ONE-WAY CARRIER SYSTEM}

In a one-way (inbound only) system, readings bubble up from end devices (i.e., meters), through the communication infrastructure, to a master station which publishes the readings.

A one-way system might be lower-cost than a two-way system but also is difficult to reconfigure should the operating environment change (Dickinson and Nicholson, 1997).
Two-way carrier system: In a two-way system (supporting both outbound and inbound), commands can be broadcast out from the master station to end devices (meters) allowing for reconfiguration of the network or to obtain readings or to convey messages etc. The device at the end of the network may then respond (inbound) with a message that carries the desired value. Outbound messages injected at a utility substation will propagate to all points downstream. This type of broadcast allows the communication system to simultaneously reach many thousands of devices all of which are known to have power and have been previously identified as candidates for load shed (Dickinson and Nicholson, 1997).

\section{COMMUNICATION CHANNEL}

A communication channel is defined as the physical path between two communication nodes on which the communication signal is propagated (Anderson, 1998; Deinzer and Stoger, 1999). The quality is estimated from how good the communication is on a channel. The quality is mostly a parameter of the noise level at the receiver and the attenuation of the electrical signal at different frequencies. The higher the noise level the harder it is to detect the received signal. If the signal gets attenuated on its way to the receiver it could also make the decision harder because the signal gets more hidden by the noise. On the power-line, the noise is generated from all loads connected to the grid (Brown, 1998).

Also, broadcast radio interferes with the communication. The attenuation is a parameter of the physical length of the channel and impedance mismatches in the grid. The power-line is often considered a harsh environment because of the time-variant characteristics of the noise and the attenuation but this is also the case in most communication systems and only limits the performance that can be achieved. Advanced communication systems exists today, designed to overcome the problems with such channels as e.g., GSM. The characteristics of the power-line channel are further described.

\section{DIGITAL COMMUNICATIONS}

In this study, some preliminaries from digital communications are critically examined. A model of a digital communication system is given in the study.

System model: Figure 1 shows a simplified model of a digital communication system. Recommended textbooks 


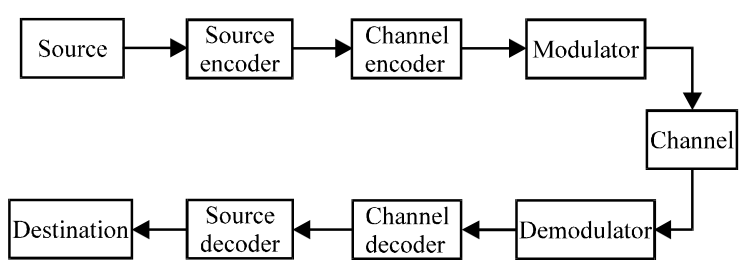

Fig. 1: A model of a digital communication system (Newbury and Morris, 1999)

on this subject are (Anderson, 1998; Brown, 1997, 1998). The objective of the communication system is to communicate digital information (a sequence of binary information digits) over a noisy channel at as high bit rates as possible. The data to be transmitted could origin from any source of information. In case the information is an analog signal such as speech then an $\mathrm{A} / \mathrm{D}$ converter must precede the transmitter. The source encoder outputs data that are to be transmitted over the channel at a certain information bit rate, $\mathrm{Rb}$. As a measure of performance we define the bit error probability $\mathrm{Pb}$ as the probability that a bit is incorrectly received at the destination. As we will see later, the channel may interfere with the communication thus increasing the bit error probability.

Source coding: Most data contains redundancy which makes it possible to compress the data. This is done by the source encoder and minimizes the amount of bits transmitted over the channel. At the receiver the source decoder unpacks the data to either an exact replica of the source (lossless data compression) or a distorted version (lossy data compression). If the received sequence does not have to be an exact copy of the transmitted stream then the degree of compression can be increased.

Channel coding: In order to reduce the bit error probability the channel encoder adds redundancy (extra control bits) to the bit sequence in a controlled way. When an error appears in the bit stream the extra information may be used by the channel decoder, to detect, and possibly correct, the error. The redundancy added is depending on the amount of correction needed but is also tuned to the characteristics of the channel. Two coding techniques often used are block codes (Burr and Brown, 1999) convolutional code (Darnell and Pem, 1999).

Modulator: The modulator produces an informationcarrying signal, propagating over the channel. At this stage the data is converted from a stream of bits into an analog signal that the channel can handle. The modulator has a set of analog waveforms at its disposal and maps a certain waveform to a binary digit or a sequence of digits. At the receiver, the demodulator tries to detect which waveform was transmitted and convert the analog information back to a sequence of bits.

Several modulation techniques exists at this stage e.g., spread-spectrum, OFDM (Orthogonal Frequency Division Multiplex), GMSK (Gaussian Minimum Shift Keying), FSK (Frequency Shift Keying), PSK (Phase Shift Keying) and QAM (Quadrature Amplitude Modulation).

Channel: The channel might be any physical medium such as coaxial cable, air, water or telephone wires. It is important to know the characteristics of the channel such as the attenuation and the noise level because these parameters directly affect the performance of the communication system.

Bandwidth: The frequency content of the information carrying signal is of great importance. The frequency interval used by the communication system is called bandwidth, W (Brown, 1998). For a specific communication method, the bandwidth needed is proportional to the bit rate. Thus, a higher bit rate needs a larger bandwidth for a fixed method. If the bandwidth is doubled then the bit rate is also doubled. In today's environment bandwidth is a limited and precious resource and the bandwidth is often constrained to a certain small interval.

This puts a restriction on the communication system to communicate within the assigned bandwidth. Today, an advanced telephone modem can achieve a bit rate of $56.6 \mathrm{~kb} \mathrm{sec}^{-1}$ using a bandwidth of $4 \mathrm{kHz}$ and the bandwidth efficiency is $14.15 \mathrm{bsec} \mathrm{Hz}{ }^{-1}$. A meter reading system for the power-line channel that has a bit rate of $10 \mathrm{~kb} \mathrm{sec}^{-1}$ and communicates within the CENELEC device. A band has a bandwidth efficiency of $0.11 \mathrm{~b} / \mathrm{sec} /$ $\mathrm{Hz}$ thus the performance of the telephone modem is much higher.

Bandwidth limitations: As described the bandwidth is proportional to the bit rate thus a large bandwidth is needed in order to communicate with high bit rates.

Signal to noise ratio: A key parameter when estimating the performance of a communication system is the Signal to Noise power Ratio (SNR) (Darnell and Pem, 1999): 


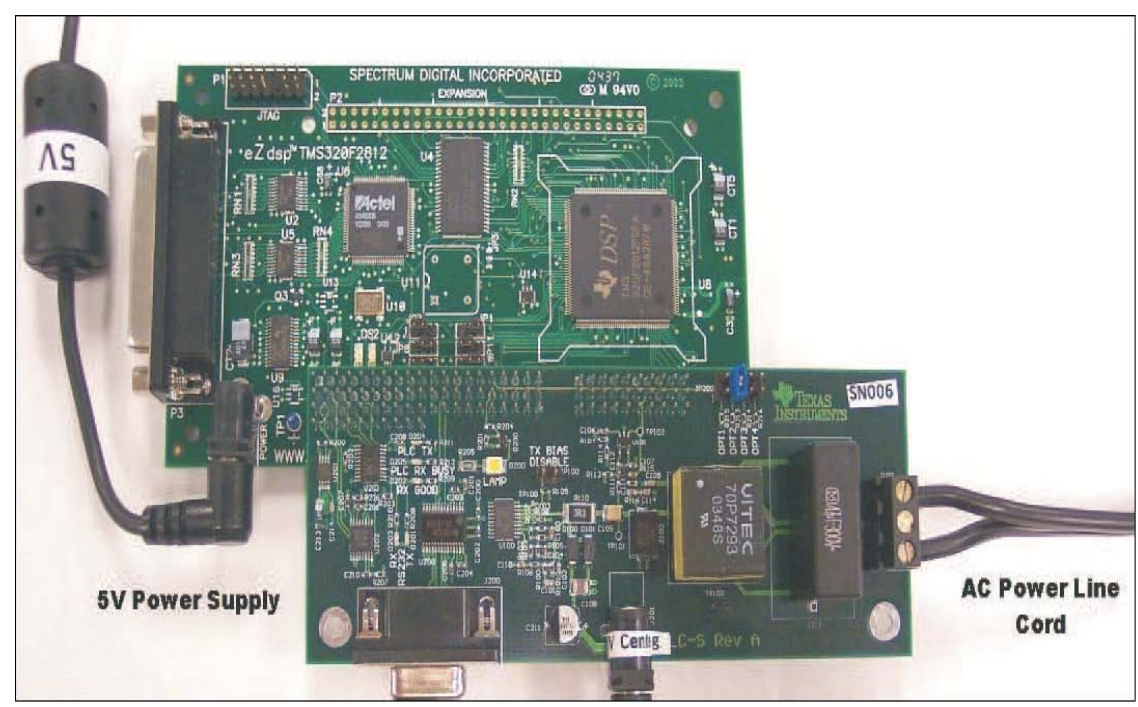

Fig. 2: Controller Based Power Line Modem Hardware (Bostoen and van de Wiel, 2000)

$$
\mathrm{SNR}=\frac{\text { Received power }}{\text { Noise power }}
$$

This parameter is related to the performance of a communication system. The higher the SNR, the better the communication links of the system. The noise power on the power-line is a sum of many different disturbances. Loads connected to the grid such as TV, computers and vacuum cleaners generate noise propagating over the power-line.

Other communication systems might also disturb the communication thus introducing noise at the receiver. Noise measurements would be found in various research articles but not limited to the following: Arzbergerl et al. (1997), Brown (1998), Burr et al. (1998), Burr and Brown (1999), Darnell and Pem (1999), Deinzer and Stoger (1999) and Dickinson and Nicholson (1997).

When the signal is propagating from the transmitter to the receiver the signal gets attenuated. If the attenuation is very high the received power gets very low and might not be detected.

The attenuation on the power-line has shown to be very high (up to $100 \mathrm{~dB}$ ) and puts a restriction on the distance from the transmitter to the receiver (Barnes, 1998; Stutt et al., 1994; Newbury and Morris, 1999). An option might be to use repeaters in the cable-boxes thus increasing the communication length.

The use of filters could improve the signal-to-noise ratio (Hideki et al., 1999). If a filter is placed at each household blocking the noise generated indoors from entering the grid, the noise level in the grid will decrease but the cost is a higher complexity. It is important to point out that although the power-line is considered a harsh environment when it comes to attenuation and disturbances, these parameters exists in any communication system used today.

An example of a designed modem is shown in Fig. 2. In effect, every electrical outlet becomes an Ethernet jack or network connection point without adding any new wires.

The power-line communication channel can in general, be modeled as having a time-varying frequency dependent signal-to-noise ratio over the communication bandwidth.

The effect of non-white Gaussian noise on different receiver structures is studied, one ideal and one sub-optimal and the importance of diversity (in frequency) is illustrated when the set of transmitter waveforms is fixed.

Researchers investigate robust, low-complexity and modulation methods which are able to handle unknown phase and attenuation which simplifies the implementation of the receiver (Fig. 3).

More significantly, the description of a communication strategy that eventually could be used for information transfer over the power-line communication channel.

In doing this, researchers combine coding, frequency diversity and the use of sub-channels (similar to Orthogonal Frequency Division Multiplex). This is a flexible structure which can be upgraded and adapted to future needs. 


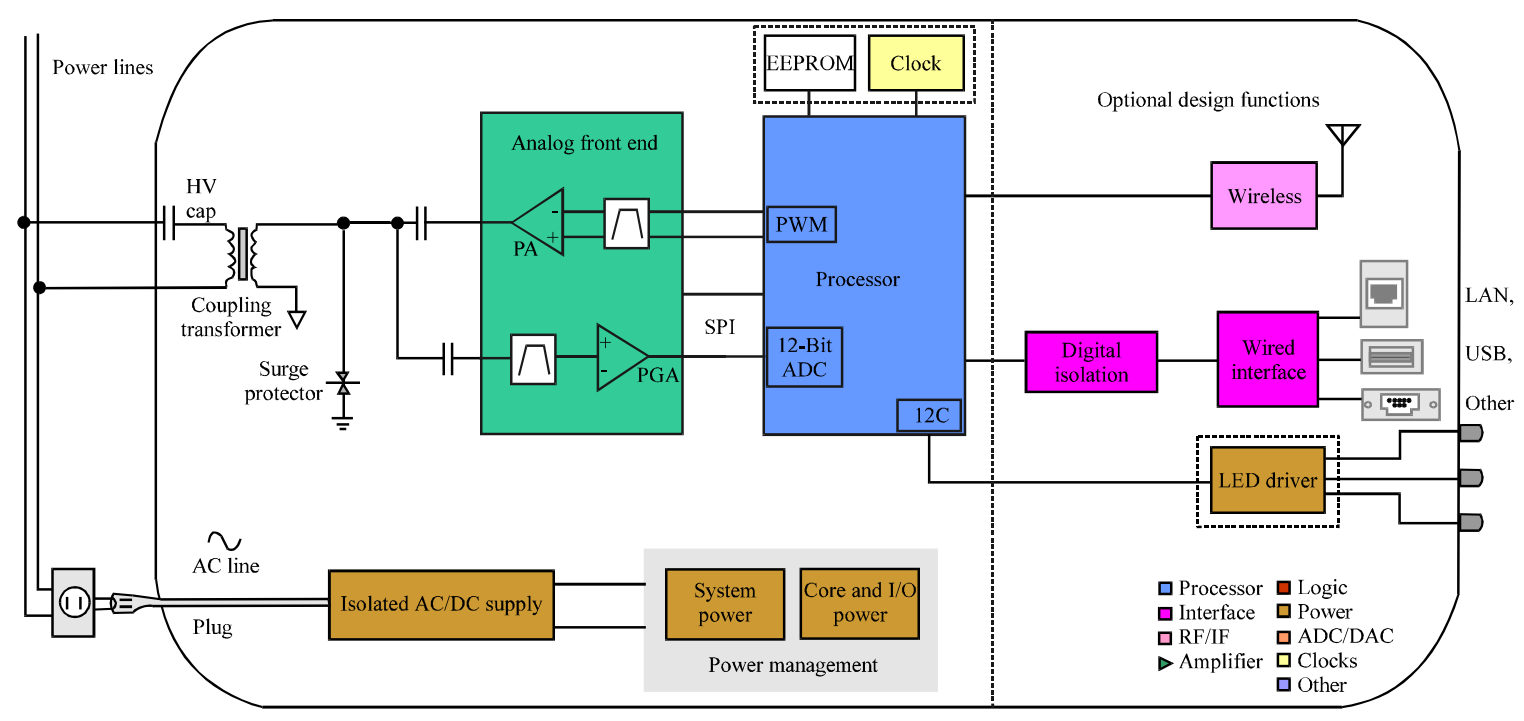

Fig. 3: A schematic diagram illustrating the components found in various systems of power line communication (Ferreiro et al., 2003)

\section{IMPORTANCE OF POWER LINE COMMUNICATION (PLC)}

Power Line Communication (PLC) solves real world connectivity problems mainly in four situations.

As a complement to wireless: Over the past few years, wireless or WiFi networks have soared in popularity. These environments give mobile users the freedom they need. But today's wireless technology also has definite limits as anyone who's ever wandered out of range with a laptop can attest. Dead zones, interference and range limits prevent full mobility and frustrate many consumers. In-home, power line communication can be used to extend the range of wireless networks by adding WiFi access points in areas with dead spots or weak coverage or by allowing a single access point to be placed in the ideal location in the house that will permit whole-house WiFi coverage. PLC essentially serves as the network backbone, allowing WiFi access points to be placed at the best coverage points in the house. By blending Power Line Communication (PLC) and $\mathrm{WiFi}$, consumers get the best of both i.e., mobility where it's needed plus reliable whole-house connectivity.

As an alternative to wireless: Although, wireless offers great benefits where mobility is required, wireless technology is seldom the best choice for connecting products that don't need mobility. Compared to wireless, Power-line technology is far easier to set up and use and it provides better range and more reliable whole house coverage. In-home, PLC is particularly useful for applications that need real time delivery of streaming audio and video or low-latency connectivity (such as VoIP telephony).

As an alternative to new wiring: Considering an option of installing PLC, running new wires to create a home network, installing a cables or even DSL modem can be expensive and disruptive. With power-line technology, the existing wires in the house serve as the network. In-home, PLC essentially lights up these wires, enabling them to serve as a virtual network that turns every electrical outlet into an Ethernet port or other network connection point.

As part of a system of home connectivity: Increasingly, industry participants are recognizing that the best way of achieving reliable whole-house connectivity is to use a mix of networking technologies that best fit the needs of a particular home.

Power line communications can serve as a primary or complementary networking technology. As a complementary technology, PLC offers a simple and costeffective method of extending the reach or optimizing the placement of WiFi or UWB access points. In this application, PLC serves as the network backbone (just as Ethernet does in enterprise settings) and wireless provides mobility for in-room requirements. PLC also works well in hybrid networks, delivering reliable bandwidth for devices that must be plugged in anyway and leaving the wireless network free to serve mobile 
devices. In addition, PLC complements other wire line networks by providing coverage where cable and telephone outlets are not available.

\section{COST EFFECTIVENESS OF POWERLINE MEDIA FOR NETWORKING DEVICES}

Power line communication uses the existing power lines within a home, building or an outdoor power distribution network to transmit data from one device to another. With a well-designed power line solution, devices should be able to communicate using the existing wiring infrastructure without any rewiring or modification. This makes power line communication one of the most cost-effective means for networking devices.

\section{APPLICATIONS THAT CAN BE ENABLED BY COMMUNICATIONS OVER POWER LINES}

Any electrical devices connected to the power line can be networked to communicate with each other. Some examples of applications are included:

Intelligent electricity meters: This solution enables utilities to network all of their electricity meters and to read them from a remote central location (automated meter reading). A power line smart transceiver-based meter can also enable utilities to remotely switch on/off power to a facility as well as detect any tampering of meters or unauthorized power consumption. Echelon's power line technology is currently being deployed in 27 million meters in Italy by their largest utility: ENEL.
Networked home appliances: Every device in a home can now communicate with each other as well as with the local electricity meter. These devices could include the refrigerator, washer/dryer, AC/heating, lighting system, security system and pool heating, etc.

As a result, utilities and consumers can monitor and manage power consumption more effectively (demand side management) thereby increasing cost savings and convenience.

\section{CHALLENGES ASSOCIATED WITH COMMUNICATING OVER POWER LINES}

Power lines were designed to carry power and not data. This means it takes a very sophisticated transceiver to reliably communicate over power lines. Many electrical devices connected to the power lines adversely impact the data that is being transmitted. The quality of the signal that is transmitted over power lines is dependent on the number and type of the electrical devices (televisions, computers and hair dryers, etc.) connected to the power lines and switched on at any given time. The quality of the signal is also dependent upon the wiring distance (not physical distance) between the transmitter and the receiver as well as the topology (wiring architecture) of the power line infrastructure in the home/building.

All of the above impediments could vary between buildings, neighborhoods and the power grids in various countries making a universal solution even more challenging. PLC devices work best when they have direct and separate access to the $\mathrm{AC}$ line as shown in Fig. 4.

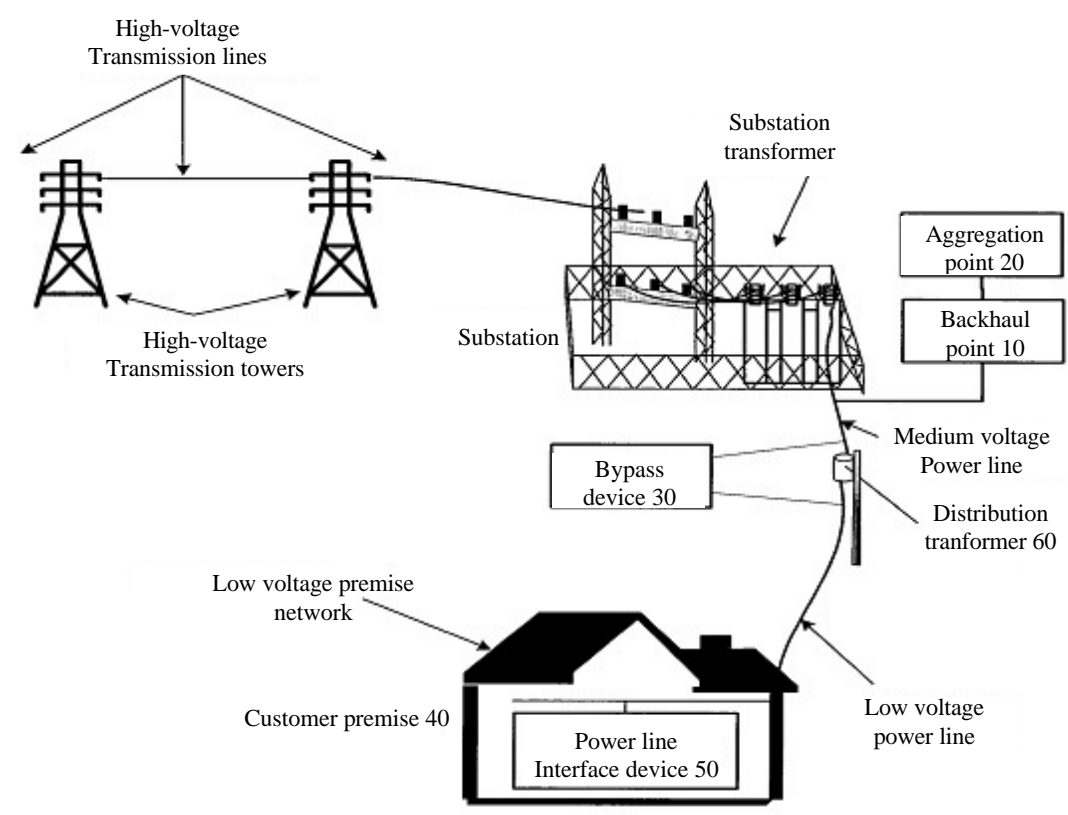

Fig. 4: Various sections of power line communication (Barnes, 1998) 


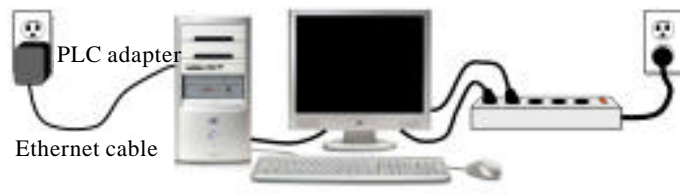

Fig. 5: Good separation with separate outlet for PLC wall adapter (Liu et al., 2001)

The implication here is that the device should not be plugged into an $\mathrm{AC}$ outlet or power strip that powers other devices such as $\mathrm{PCs}$, printers and monitors. The use of the power strip provides some distancing between the PLC device and other devices that may act as noise sources. Most power strips have surge suppression built in but the better ones also have EMI filtering which works to further isolate equipment noise from the line. One of two outlets even on the same wall may offer some improvement in performance (Fig. 5).

\section{CONCLUSION}

This study then focuses on the effectiveness and usefulness of an alien means of communication, Power Line Communication (PLC). The main advantage with power-line communication is the use of an existing infrastructure. Wires exist to every household connected to the power-line network.

\section{REFERENCES}

Anderson, J.B., 1998. Digital Transmission Engineering. IEEE Press, New York, USA.

Arzbergerl, M., K. Dostert, T. Waldeck and M. Zimmermann, 1997. Fundamental properties of the low voltage power distribution grid. Proceedings of the International Symposium on Power-Line Communications and its Applications, April 2-4, 1997, Saalbau, Essen, Germany, pp: 45-50.

Barnes, J.S., 1998. A physical multi-path model for power distribution network propagation. Proceedings of the International Symposium on Power-line Communications and its Applications, March 24-26, 1998, Tokyo, Japan, pp: 76-89.

Bostoen, T. and O. van de Wiel, 2000. Modeling the low-voltage power distribution network in the frequency band from $0.5 \mathrm{MHz}$ to $30 \mathrm{MHz}$ for broadband Powerline Communications (PLC). Proceedings of the International Zurich Seminar on Broadband Communications, February 15-17, 2000, Zurich, Switzerland, pp: 171-178.

Brown, P., 1997. Directional coupling of high frequency signals onto power networks. Proceedings of the International Symposium on Power-Line Communications and its Applications, April 2-4, 1997, Essen, Germany, pp: 19-24.
Brown, P.A., 1998. Some key factors influencing data transmission rates in the power line environment when utilising carrier frequencies above $1 \mathrm{MHz}$. Proceedings of the International Symposium on Power-Line Communications and its Applications, April 1998, Tokyo, Japan, pp: 67-75.

Burr, A.G. and P.A. Brown, 1999. Application of OFDM to powerline telecommunications. Proceedings of the 3rd International Symposium on Power-Line Communications and its Applications, March 30-April 1, 1999, Lancaster, UK.

Burr, A.G., D.M.W. Reed and P.A. Brown, 1998. HF broadcast interference on LV mains distribution networks. Proceedings of the International Symposium on Power-Line Communications and its Applications, March 1998, Tokyo, Japan, pp: 253-262.

Darnell, M. and N.D. Pem, 1999. OFDM using complementary sequences for data transmissionover non-gaussian channel. Proceedings of the 3rd International Symposium on Power-Line Communications and its Applications, March 30-April 1, 1999, Lancaster, UK., pp: 147-156.

Deinzer, M. and M. Stoger, 1999. Integrated PLC-modem based on OFDM. Proceedings of the 3rd International Symposium on Power-Line Communications and its Applications, March 30April 1, 1999, Lancaster, UK.

Dickinson, J. and P. Nicholson, 1997. Calculating the high frequency transmission line parameters of power cables. Proceedings of the International Symposium on Power-Line Communications and its Applications, April 2-4, 1997, Essen, Germany, pp: $127-133$.

Ferreiro, M., M. Cacheda and C. Mosquera, 2003. A low complexity all-digital DS-SS transceiver for powerline communications. Proceedings of the 7 th International Symposium on Power-Line Communications and Its Applications, March 26-28, 2003, Kyoto, Japan, pp: 286-291.

Hideki, K., M. Shin'ichi and M. Norihiko, 1999. Parameter estimation and error rate performance of optimum receiver under Class-A impulsive radio environment. IEICE Trans. Commun., J82-B: 2364-2374.

Liu, C.H., E. Wade and H.H. Asada, 2001 . Reduced-cable smart motors using DC power line communication. Proc. IEEE Int. Conf. Robotics Autom., 4: 3831-3838.

Newbury, J.E. and K.J. Morris, 1999. Power line carrier systems for industrial control applications. IEEE Trans. Power Delivery, 14: 1191-1196.

Stutt, C.A., W.C. Hughes, R.C. Rustay and J.T. Gajjar, 1994. Experimental verification on distribution feeder PLC propagation model computations. IEEE Trans. Power Delivery, 9: 519-524. 